\title{
GROUP FACTORISATIONS, UNIFORM AUTOMORPHISMS, AND PERMUTATION GROUPS OF SIMPLE DIAGONAL TYPE
}

\author{
CHERYL E. PRAEGER AND CSABA SCHNEIDER
}

\begin{abstract}
We present a new proof, which is independent of the finite simple group classification and applies also to infinite groups, that quasiprimitive permutation groups of simple diagonal type cannot be embedded into wreath products in product action. The proof uses several deep results that concern factorisations of direct products involving subdirect subgroups. We find that such factorisations are controlled by the existence of uniform automorphisms.
\end{abstract}

\section{INTRODUCTION}

The O'Nan-Scott Theorem for finite primitive and quasiprimitive permutation groups identifies several classes of such groups and claims that each primitive or quasiprimitive group is a member of a unique class. In several combinatorial and group theoretic applications, it is necessary to understand the possible inclusions among primitive and quasiprimitive permutation groups. Such inclusions were studied by the first author for finite primitive permutation groups in [Pra90], while inclusions of finite quasiprimitive groups in primitive groups were described by R. W. Baddeley and the first author in [BP03]. The possible inclusions are described in both cases by considering separately each of the O'Nan-Scott types of these permutation groups. Some of these results rely on the finite simple group classification (FSGC). In this paper we show how to remove the assumption of finiteness and hence the dependence on the FSGC for embeddings of simple diagonal type groups into wreath products in product action (see Section 4 for the definitions).

It was proved in [Pra90] that a finite primitive group of simple diagonal type cannot be a subgroup of a wreath product in product action and a similar result was proved in [BPS06, Corollary 1.3] for finite quasiprimitive groups of simple diagonal type. The latter theorem formed an important part of the description of primitive overgroups of finite quasiprimitive permutation groups in [BP03]. As with many results concerning the

Date: November 1, 2018.

2010 Mathematics Subject Classification. 20B05,20B35,20E22.

We are grateful to Robert Guralnick and Kay Magaard for their help with the construction in Example 2.2. We thank Pavel Shumyatsky for his advice on the literature on uniform automorphisms. The first author wishes to acknowledge the support of the Australian Research Council Discovery Grant (DP160102323). The second author was supported by the research projects 302660/2013-5 (CNPq, Produtividade em Pesquisa), 475399/2013-7 (CNPq, Universal), and the APQ-00452-13 (Fapemig, Universal). 
inclusion problem, [BPS06, Corollary 1.3] depended on the FSGC. As mentioned above, we extend this result to infinite permutation groups of simple diagonal type and prove that such groups cannot be embedded into primitive groups of product action type.

Theorem 1.1. Suppose that $G$ is a quasiprimitive permutation group of simple diagonal type acting on a possibly infinite set $\Omega$. Then $G$ is not contained in a subgroup of Sym $\Omega$ which is permutationally isomorphic to $\operatorname{Sym} \Gamma \imath \mathrm{S}_{\ell}$ with $|\Gamma| \geqslant 2$ and $\ell \geqslant 2$ acting in its product action.

As is typical in the study of the inclusion problem, the key result underpinning the proof of Theorem 1.1 in the finite case is a factorisation result [BP03, Lemma 2.2] concerning factorisations of finite characteristically simple groups using subdirect subgroups as factors. The proof of [BP03, Lemma 2.2] depends on the fact that a finite simple group does not admit uniform automorphisms, which, in turn, is one of the well-known consequences of the FSGC. We noticed that this result can be extended to arbitrary groups that do not admit a uniform automorphism; see Section 2 for the definition of a uniform automorphism and of a strip.

Theorem 1.2. Suppose that $T$ is a group that does not admit a uniform automorphism and that $X, Y$ are direct products of pairwise disjoint non-trivial full strips in $T^{k}$ with $k \geqslant 2$. Then $X Y \neq T^{k}$.

The proof of Theorem 1.1 relies on the technical Proposition 3.1, and, in turn, the proof of Proposition 3.1 uses Theorem 1.2.

The O'Nan-Scott Theorem for finite primitive groups was originally intended to describe the maximal subgroups of finite symmetric groups; see [Sco80, LPS87]. Along the same lines, some maximal subgroups of infinite symmetric groups have been associated with structures such as subsets, partitions [B+94], and cartesian decompositions [CMM96]. One class of maximal subgroups of a finite symmetric group is formed by maximal subgroups of simple diagonal type. It would be interesting to explore if infinite symmetric groups also have maximal subgroups that are associated (via, for instance, filters or ideals, as in $[\mathrm{B}+94, \mathrm{CMM} 96])$ to groups of simple diagonal type. Theorem 1.1 suggests that none of the maximal subgroups considered in [B+94, CMM96] contain a primitive group of simple diagonal type. On the other hand, simple diagonal type groups do lie in maximal subgroups of $\operatorname{Sym} \Omega$, at least when $\Omega$ is countable by [MP90, Theorem 1.1].

It is a curious fact that the results presented in this note have two distinct points of connection with the work of our late friend and colleague, Laci Kovács. As early as the 1960's Laci studied uniform automorphisms for solvable groups (see for instance [Kov88a]), and in the 1980's, his attention turned towards the theory of primitive permutation groups. He devoted an entire paper [Kov88b] to primitive groups of simple diagonal type, and it was this paper in which the terminology 'simple diagonal type' was first used. 


\section{UNIFORM AUTOMORPHISMS AND FACTORISATIONS OF DIRECT PRODUCTS}

In this section we prove several results on factorisations of direct products using diagonal subgroups.

An automorphism $\alpha$ of a group $G$ is uniform, if the map $\tilde{\alpha}: g \mapsto g^{-1}(g \alpha)$ is surjective. If $G$ is a finite group, then a map $G \rightarrow G$ is surjective if and only if it is injective. Thus, if $G$ is a finite group, then $\alpha \in$ Aut $G$ is not uniform if and only if the map $g \mapsto g^{-1}(g \alpha)$ is not injective; that is, $g^{-1}(g \alpha)=h^{-1}(h \alpha)$ for some distinct $g, h \in G$. The last equation is equivalent to $h g^{-1}=\left(h g^{-1}\right) \alpha$; that is, in this case, the element $h g^{-1}$ is a non-identity fixedpoint of the automorphism $\alpha$. It is a consequence of the FSGC that every automorphism of a non-abelian finite simple group has non-identity fixed points [Gor13, Theorem 1.48]. In fact the following stronger result holds; see [KS06, 9.5.3] for a proof.

Lemma 2.1. A finite non-solvable group has no uniform (that is, fixed-point-free) automorphisms.

The situation is rather different for the class of infinite simple groups.

Example 2.2. Let $\mathbb{F}$ be the algebraic closure of the finite field $\mathbb{F}_{p}$ and consider the group $G=\mathrm{SL}_{n}(\mathbb{F})$ with $n \geqslant 2$. Then $G$ is a connected algebraic group (see [Spr98, Exercise 2.2.2]). Further, the $p$-th powering map $\left(a_{i, j}\right) \mapsto\left(a_{i, j}^{p}\right)$ defines an automorphism $\varphi: G \rightarrow G$ known as the Frobenius automorphism. By Lang's Theorem [Spr98, Theorem 4.4.17], the map $G \rightarrow G, g \mapsto g^{-1}(g \varphi)$ is surjective. Since the centre $\mathrm{Z}(G)$ is invariant under automorphisms of $G, \varphi$ induces an automorphism $\bar{\varphi}$ of the infinite simple group $\mathrm{PSL}_{n}(\mathbb{F}) \cong G / Z(G)$ such that the map $\mathrm{PSL}_{n}(\mathbb{F}) \rightarrow \mathrm{PSL}_{n}(\mathbb{F})$ defined as $g \mapsto g^{-1}(g \bar{\varphi})$ is surjective. Thus $\bar{\varphi}$ is a uniform automorphism of the infinite simple group $\mathrm{PSL}_{n}(\mathbb{F})$. Similar examples can be constructed using other connected algebraic groups of Lie type.

Suppose that $G$ is a direct product $G=G_{1} \times \cdots \times G_{k}$ of groups $G_{i}$ and, for $i=1, \ldots, k$, let $\sigma_{i}: G \rightarrow G_{i}$ be the coordinate projection. A subgroup $H$ of $G$ is said to be a strip if, for all $i, H \sigma_{i} \cong H$ or $H \sigma_{i}=1$. If $H$ is a strip, then $H$ is a diagonal subgroup in the sense that there exist indices $1 \leqslant i_{1}<\cdots<i_{m} \leqslant k$, a subgroup $H_{0} \leqslant G_{i_{1}}$ and, for $j \in\{2, \ldots, m\}$, injective homomorphisms $\alpha_{j}: H_{0} \rightarrow G_{i_{j}}$ such that

$$
\begin{aligned}
H= & \left\{\left(g_{1}, \ldots, g_{k}\right) \mid g_{i_{1}} \in H_{0}, g_{i_{j}}=g_{i_{1}} \alpha_{j} \text { for } 2 \leqslant j \leqslant m\right. \text { and } \\
& \left.g_{i}=1 \text { if } i \notin\left\{i_{1}, \ldots, i_{m}\right\}\right\} .
\end{aligned}
$$

The set $\left\{G_{i_{1}}, \ldots, G_{i_{m}}\right\}$ is said to be the support of the strip $H$ and is denoted by Supp $H$. The strip $H$ is said to be non-trivial, if $|\operatorname{Supp} H| \geqslant 2$, and it is said to be full if $H \sigma_{i}=G_{i}$ whenever $G_{i} \in \operatorname{Supp} H$. Thus, if $H$, written as in (1), is a full strip, then $H_{0}=G_{i_{1}}$ and the $\alpha_{j}$ are isomorphisms. In particular, the $G_{i_{j}}$ are pairwise isomorphic for $j=1, \ldots, m$.

The existence of factorisations of direct products with strips as factors is related to the existence of uniform automorphisms. It was proved in [BP03, Lemma 2.2] that a finite direct power of a finite simple group cannot be factorised into a product of two subgroups both of which are direct products of non-trivial full strips. We generalise this result for a larger class of groups. We start by proving the following lemma for two factors. 
Lemma 2.3. The following assertions are equivalent for a group $G$.

(1) There exist non-trivial full strips $X$ and $Y$ in $G \times G$ such that $G \times G=X Y$.

(2) $G$ admits a uniform automorphism.

Proof. Suppose that $G \times G=X Y$ with $X$ and $Y$ non-trivial full strips of $G \times G$. Then there exist $\alpha, \beta \in$ Aut $G$ such that $X=\{(g, g \alpha) \mid g \in G\}$ and $Y=\{(g, g \beta) \mid g \in G\}$. If $g \in G$, then there exist $g_{1}, g_{2} \in G$, such that $(g, 1)=\left(g_{1}^{-1}, g_{1}^{-1} \alpha\right)\left(g_{2}, g_{2} \beta\right)$. Thus $g=g_{1}^{-1} g_{2}$ and $1=\left(g_{1}^{-1} \alpha\right)\left(g_{2} \beta\right)$, and so $g_{2}=g_{1} \alpha \beta^{-1}$. Hence $g=g_{1}^{-1}\left(g_{1} \alpha \beta^{-1}\right)$, which implies that the map $x \mapsto x^{-1}\left(x \alpha \beta^{-1}\right)$ is surjective. That is, the automorphism $\alpha \beta^{-1}$ is uniform.

Conversely, assume that $\alpha \in$ Aut $G$ is uniform. Set $X=\{(g, g) \mid g \in G\}$ and $Y=\{(g, g \alpha) \mid g \in G\}$. Let $(x, y) \in G \times G$. Choose $h \in G$ such that $h^{-1}(h \alpha)=x^{-1} y$ (such an $h$ exists, as $\alpha$ is uniform) and let $g=x h^{-1}$. Then $g(h \alpha)=x h^{-1}(h \alpha)=y$ and $g h=x$. Thus $(g, g)(h, h \alpha)=(x, y)$. Therefore $X Y=G \times G$.

Finite groups with uniform automorphisms do exist. For example, the automorphism $\alpha: x \mapsto x^{-1}$ of a finite abelian group $G$ of odd order is uniform. In this case, we do in fact obtain a factorisation

$$
G \times G=\{(g, g) \mid g \in G\}\{(g, g \alpha) \mid g \in G\} .
$$

Lemma 2.4. Suppose that $T$ is a group and, for $i=1, \ldots, d$, let $\alpha_{i}, \beta_{i} \in$ Aut $T$. Consider the following subgroups $X$ and $Y$ of $T^{2 d}$ :

$$
\begin{aligned}
X & =\left\{\left(t_{1}, t_{1} \alpha_{1}, t_{2}, t_{2} \alpha_{2} \ldots, t_{d}, t_{d} \alpha_{d}\right) \mid t_{i}=T\right\} \text { and } \\
Y & =\left\{\left(s_{d} \beta_{d}, s_{1}, s_{1} \beta_{1}, s_{2}, s_{2} \beta_{2}, \ldots, s_{d-1}, s_{d-1} \beta_{d-1}, s_{d}\right) \mid s_{i} \in T\right\}
\end{aligned}
$$

Then the following are equivalent:

(1) $X Y=T^{2 d}$;

(2) the automorphism $\alpha_{1} \beta_{1} \alpha_{2} \beta_{2} \cdots \alpha_{d} \beta_{d}$ of $T$ is uniform.

Proof. Assume that $X Y=T^{2 d}$ and let $x \in T$. Then there exist

$$
\begin{aligned}
\left(t_{1}^{-1}, t_{1}^{-1} \alpha_{1}, t_{2}^{-1}, t_{2}^{-1} \alpha_{2}, \ldots, t_{d}^{-1}, t_{d}^{-1} \alpha_{d}\right) & \in X \text { and } \\
\left(s_{d} \beta_{d}, s_{1}, s_{1} \beta_{1}, \ldots, s_{d-1}, s_{d-1} \beta_{d-1}, s_{d}\right) & \in Y
\end{aligned}
$$

such that $(x, 1, \ldots, 1)$ is equal to

$$
\left(t_{1}^{-1}, t_{1}^{-1} \alpha_{1}, t_{2}^{-1}, t_{2}^{-1} \alpha_{2}, \ldots, t_{d}^{-1}, t_{d}^{-1} \alpha_{d}\right) \cdot\left(s_{d} \beta_{d}, s_{1}, s_{1} \beta_{1}, \ldots, s_{d-1}, s_{d-1} \beta_{d-1}, s_{d}\right)
$$


Comparing the entries for these two expressions, we obtain that

$$
\begin{aligned}
x & =t_{1}^{-1}\left(s_{d} \beta_{d}\right) \\
t_{1} & =s_{1} \alpha_{1}^{-1} \\
t_{2} & =s_{1} \beta_{1} \\
t_{2} & =s_{2} \alpha_{2}^{-1} \\
& \vdots \\
t_{d} & =s_{d-1} \beta_{d-1} \\
t_{d} & =s_{d} \alpha_{d}^{-1} .
\end{aligned}
$$

Hence $s_{i}=s_{i-1} \beta_{i-1} \alpha_{i}$ for $i=2, \ldots, d$. Thus, by induction, $s_{d}=s_{1} \beta_{1} \alpha_{2} \cdots \beta_{d-1} \alpha_{d}$. Then setting $s=s_{1} \alpha_{1}^{-1}$ and using the first two equations in the system above, we obtain

$$
x=\left(s_{1} \alpha_{1}^{-1}\right)^{-1}\left(s_{1} \beta_{1} \alpha_{2} \ldots \beta_{d-1} \alpha_{d} \beta_{d}\right)=s^{-1}\left(s \alpha_{1} \beta_{1} \ldots \alpha_{d} \beta_{d}\right) .
$$

Since we chose $x \in T$ arbitrarily, the automorphism $\alpha_{1} \beta_{1} \ldots \alpha_{d} \beta_{d}$ of $T$ is uniform.

Conversely, assume that $\alpha:=\alpha_{1} \beta_{1} \ldots \alpha_{d} \beta_{d}$ is a uniform automorphism of $T$. Let $x=\left(x_{1}, \ldots, x_{2 d}\right)$ be an arbitrary element of $T^{2 d}$. Since $\alpha$ is uniform, there exists $s_{0} \in T$ such that

$$
s_{0}^{-1}\left(s_{0} \alpha\right)=\prod_{i=d}^{1}\left(\left(x_{2 i}^{-1} \beta_{i} \alpha_{i+1} \cdots \alpha_{d} \beta_{d}\right)\left(x_{2 i-1} \alpha_{i} \beta_{i} \cdots \alpha_{d} \beta_{d}\right)\right) .
$$

Let $s_{d}=s_{0} \beta_{d}^{-1}$ and $t_{d}=\left(s_{d} x_{2 d}^{-1}\right) \alpha_{d}^{-1}$. We next define sequence

$$
s_{d-1}, t_{d-1}, s_{d-2}, \ldots, s_{1}, t_{1}
$$

'backwards recursively' in the sense that we define, for $i=d-1, \ldots, 1$, the elements $s_{i}$ and $t_{i}$ assuming that we have defined the elements $s_{i+1}$ and $t_{i+1}$ :

$$
\begin{aligned}
s_{i} & =\left(t_{i+1} x_{2 i+1}\right) \beta_{i}^{-1} \\
t_{i} & =\left(s_{i} x_{2 i}^{-1}\right) \alpha_{i}^{-1}
\end{aligned}
$$

Now let

$$
\begin{aligned}
t & =\left(t_{1}^{-1}, t_{1}^{-1} \alpha_{1}, \ldots, t_{d}^{-1}, t_{d}^{-1} \alpha_{d}\right) \quad \text { and } \\
s & =\left(s_{d} \beta_{d}, s_{1}, s_{1} \beta_{1}, \ldots, s_{d-1}, s_{d-1} \beta_{d-1}, s_{d}\right) .
\end{aligned}
$$

We claim that $t s=x$. The definition of $t_{i}$ and $s_{i}$ implies that

$$
\begin{aligned}
x_{2 i} & =\left(t_{i}^{-1} \alpha_{i}\right) s_{i} \text { and } \\
x_{2 i+1} & =t_{i+1}^{-1}\left(s_{i} \beta_{i}\right)
\end{aligned}
$$

for all indices $2 i$ and $2 i+1$ between 2 and $2 d$. This shows that the product $t s$ agrees with $x$ from the second coordinate onwards. It remains to show that $t s$ agrees with $x$ in the 
first coordinate also. Using the equations for $t_{i}$ and $s_{i}$, we obtain by induction that

$$
\begin{array}{r}
t_{1}=\left(s_{d} \alpha_{d}^{-1} \beta_{d-1}^{-1} \cdots \beta_{1}^{-1} \alpha_{1}^{-1}\right)\left(x_{2 d}^{-1} \alpha_{d}^{-1} \beta_{d-1}^{-1} \cdots \beta_{1}^{-1} \alpha_{1}^{-1}\right) \\
\left(x_{2 d-1} \beta_{d-1}^{-1} \cdots \beta_{1}^{-1} \alpha_{1}^{-1}\right) \cdots\left(x_{2}^{-1} \alpha_{1}^{-1}\right) .
\end{array}
$$

Applying $\alpha$ to the last equation and considering the definition of $s_{d}$, we obtain

$$
\begin{aligned}
t_{1} \alpha=\left(s_{d} \beta_{d}\right)\left(x_{2 d}^{-1} \beta_{d}\right)\left(x_{2 d-1} \alpha_{d} \beta_{d}\right) \cdots\left(x_{2}^{-1} \beta_{1} \alpha_{2} \beta_{2} \cdots \alpha_{d} \beta_{d}\right)= \\
s_{0}\left(x_{2 d}^{-1} \beta_{d}\right)\left(x_{2 d-1} \alpha_{d} \beta_{d}\right) \cdots\left(x_{2}^{-1} \beta_{1} \alpha_{2} \beta_{2} \cdots \alpha_{d} \beta_{d}\right)
\end{aligned}
$$

which gives (using (2) for the last equality in the next line)

$$
\begin{aligned}
& s_{0}^{-1}\left(t_{1} \alpha\right)\left(x_{1} \alpha\right)=s_{0}^{-1}\left(t_{1} \alpha\right)\left(x_{1} \alpha_{1} \beta_{1} \alpha_{2} \beta_{2} \cdots \alpha_{d} \beta_{d}\right)= \\
& \left(x_{2 d}^{-1} \beta_{d}\right)\left(x_{2 d-1} \alpha_{d} \beta_{d}\right) \cdots\left(x_{2}^{-1} \beta_{1} \alpha_{2} \beta_{2} \cdots \alpha_{d} \beta_{d}\right)\left(x_{1} \alpha_{1} \beta_{1} \alpha_{2} \beta_{2} \cdots \alpha_{d} \beta_{d}\right)=s_{0}^{-1}\left(s_{0} \alpha\right) .
\end{aligned}
$$

This implies that $x_{1}=t_{1}^{-1} s_{0}$ which, in turn, equals $t_{1}^{-1}\left(s_{d} \beta_{d}\right)$. Therefore $t s$ agrees with $x$ in its first coordinate also, and so $t s=x$. Since the choice of the element $x$ was arbitrary, $X Y=T^{2 d}$.

If $M$ is the direct product $M=T_{1} \times \cdots \times T_{k}$ and $I$ is a subset of $\left\{T_{1}, \ldots, T_{k}\right\}$ or a subset of $\{1, \ldots, k\}$, then $\sigma_{I}$ denotes the corresponding coordinate projection from $M$ to $\prod_{T_{i} \in I} T_{i}$ or to $\prod_{i \in I} T_{i}$, respectively.

The proof of the following lemma uses some simple graph theoretic concepts. The graphs that occur in this proof are undirected graphs without multiple edges and loops. A graph which does not contain a cycle is said to be a forest, while a connected graph without a cycle is a tree. The valency of a vertex $v$ in a graph is the number of vertices that are adjacent to $v$. A vertex $v$ in a forest with valency one is said to be a leaf.

We now prove Theorem 1.2.

The proof of Theorem 1.2. By assumption, $X=X_{1} \times \cdots \times X_{r}$ and $Y=Y_{1} \times \cdots \times Y_{s}$, where the $X_{i}$ and $Y_{i}$ are non-trivial full strips. Suppose that $T^{k}=X Y$. At the end of the proof, this will lead to a contradiction.

Let $\Gamma$ be the graph with vertex set $\left\{X_{1}, \ldots, X_{r}, Y_{1}, \ldots, Y_{s}\right\}$ such that two vertices of $\Gamma$ are adjacent if and only if the supports of the strips are not disjoint. First note that two strips that belong to $X$ are disjoint, and the same is true for two strips that belong to $Y$. Hence $\Gamma$ is a bipartite graph with bipartition $\left\{X_{1}, \ldots, X_{r}\right\} \cup\left\{Y_{1}, \ldots, Y_{s}\right\}$. We prove the result by proving a series of claims. Recall that $T^{k}=X Y$. Suppose that $T_{1}, \ldots, T_{k}$ are the internal direct factors of $T^{k}$; that is $T_{1}, \ldots, T_{k}$ are normal subgroups of $M$ such that $M=T^{k}=T_{1} \times \cdots \times T_{k}$.

Claim 1. Each vertex of $\Gamma$ lies on at least one edge.

Proof of Claim 1. If, say, $X_{1}$ lies on no edge of $\Gamma$, then $\operatorname{Supp} X_{1} \cap \operatorname{Supp} Y_{i}=\emptyset$ for each $i$, and so $\operatorname{Supp} X_{1} \cap \operatorname{Supp} Y=\emptyset$. Thus if $\sigma$ is the projection of $M$ onto $\prod_{T_{i} \in \operatorname{Supp} X_{1}} T_{i}$, then $Y \sigma=1$, and so

$$
M \sigma=(X Y) \sigma=X \sigma=X_{1} \cong T
$$


contradicting the fact that $X_{1}$ is non-trivial. Thus $X_{1}$ lies on at least one edge and the same proof shows that each $X_{i}$ and each $Y_{i}$ lies on at least one edge.

Claim 2. If $X_{j_{1}}$ and $Y_{j_{2}}$ are adjacent in $\Gamma$, then $\left|\operatorname{Supp} X_{j_{1}} \cap \operatorname{Supp} Y_{j_{2}}\right|=1$.

Proof of Claim 2. This follows from Lemma 2.3. Indeed, if $T_{i_{1}}, T_{i_{2}} \in \operatorname{Supp} X_{j_{1}} \cap \operatorname{Supp} Y_{j_{2}}$ and $\sigma=\sigma_{\left\{i_{1}, i_{2}\right\}}$, then $X_{j_{1}} \sigma$ and $Y_{j_{2}} \sigma$ are non-trivial full strips in $T_{i_{1}} \times T_{i_{2}}$ such that $T_{i_{1}} \times T_{i_{2}}=(X \sigma)(Y \sigma)=\left(X_{j_{1}} \sigma\right)\left(Y_{j_{2}} \sigma\right)$. Since $T$ does not admit a uniform automorphism, this is a contradiction, by Lemma 2.3.

By Claim 2, an edge in $\Gamma$ that connects $X_{j_{1}}$ and $Y_{j_{2}}$ can be labelled with $T_{i}$ where $\left\{T_{i}\right\}=\operatorname{Supp} X_{j_{1}} \cap \operatorname{Supp} Y_{j_{2}}$.

Claim 3. $\Gamma$ does not contain a cycle.

Proof of Claim 3. Suppose to the contrary that $\Gamma$ contains a cycle, and choose a cycle $X_{1}, Y_{1}, \ldots, X_{d}, Y_{d}$ of shortest length $2 d$. Then $2 d \geqslant 4$ and the $X_{i}$ and the $Y_{i}$ are pairwise distinct. By reordering the $T_{i}$, we may also assume without loss of generality that

$$
\begin{aligned}
\left\{T_{1}\right\} & =\operatorname{Supp} Y_{d} \cap \operatorname{Supp} X_{1} ; \\
\left\{T_{2}\right\} & =\operatorname{Supp} X_{1} \cap \operatorname{Supp} Y_{1} ; \\
\left\{T_{3}\right\} & =\operatorname{Supp} Y_{1} \cap \operatorname{Supp} X_{2} ; \\
& \vdots \\
\left\{T_{2 i-1}\right\} & =\operatorname{Supp} Y_{i-1} \cap \operatorname{Supp} X_{i} ; \\
\left\{T_{2 i}\right\} & =\operatorname{Supp} X_{i} \cap \operatorname{Supp} Y_{i} ; \\
& \vdots \\
\left\{T_{2 d-1}\right\} & =\operatorname{Supp} Y_{d-1} \cap \operatorname{Supp} X_{d} ; \\
\left\{T_{2 d}\right\} & =\operatorname{Supp} X_{d} \cap \operatorname{Supp} Y_{d} .
\end{aligned}
$$

In particular, Supp $X_{i} \cap\left\{T_{1}, \ldots, T_{2 d}\right\}=\left\{T_{2 i-1}, T_{2 i}\right\}$, Supp $Y_{i} \cap\left\{T_{1}, \ldots, T_{2 d}\right\}=\left\{T_{2 i}, T_{2 i+1}\right\}$ for $i=1, \ldots, d-1$, and $\operatorname{Supp} Y_{d} \cap\left\{T_{1}, \ldots, T_{2 d}\right\}=\left\{T_{2 d}, T_{1}\right\}$. The factors $T_{2 i}$, with $i=1, \ldots, d$, are pairwise distinct, since the $\operatorname{Supp} X_{i}$ are pairwise disjoint. Similarly the factors $T_{2 i-1}$, with $i=1, \ldots, d$, are pairwise distinct since the $\operatorname{Supp} Y_{i}$ are pairwise disjoint. Suppose that $T_{2 i}=T_{2 j-1}$ where $1 \leqslant i, j \leqslant d$. Since $T_{2 j-1} \in \operatorname{Supp} X_{j}$ and $T_{2 i} \in \operatorname{Supp} X_{i}$, we have $i=j$. But then $T_{2 i-1} \in \operatorname{Supp} Y_{i-1}$ while $T_{2 i} \in \operatorname{Supp} Y_{i}$ contradicting the fact that $\operatorname{Supp} Y_{i-1} \cap \operatorname{Supp} Y_{i}=\emptyset$. Thus all the $T_{i}$ are pairwise distinct. Let $\sigma$ denote the projection $\sigma_{\{1, \ldots, 2 d\}}$. Then $X \sigma=X_{1} \sigma \times \cdots \times X_{d} \sigma$ and $Y \sigma=Y_{1} \sigma \times \cdots \times Y_{d} \sigma$. Further, for $i=1, \ldots, d, X_{i} \sigma$ is a non-trivial full strip in $T_{2 i-1} \times T_{2 i}$, for $i=1, \ldots, d-1, Y_{i} \sigma$ is a non-trivial full strip in $T_{2 i} \times T_{2 i+1}$, while $Y_{d} \sigma$ is a non-trivial full strip in $T_{2 d} \times T_{1}$. Thus there exist isomorphisms $\alpha_{i}: T_{2 i-1} \rightarrow T_{2 i}$ (for $i=1, \ldots, d$ ), $\beta_{i}: T_{2 i} \rightarrow T_{2 i+1}$ (for $i=1, \ldots, d-1)$ and $\beta_{d}: T_{2 d} \rightarrow T_{1}$ such that

$$
\begin{aligned}
& X \sigma=\left\{\left(t_{1}, t_{1} \alpha_{1}, t_{2}, t_{2} \alpha_{2}, \ldots, t_{d}, t_{d} \alpha_{d}\right) \mid t_{i} \in T_{2 i-1}\right\} \text { and } \\
& Y \sigma=\left\{\left(s_{d} \beta_{d}, s_{1}, s_{1} \beta_{1}, \ldots, s_{d-1}, s_{d-1} \beta_{d-1}, s_{d}\right) \mid s_{i} \in T_{2 i}\right\} .
\end{aligned}
$$


Since $X Y=T^{k}$, we find that $(X \sigma)(Y \sigma)=T_{1} \times \cdots \times T_{2 d}$. By Lemma 2.4, the automorphism $\alpha_{1} \beta_{1} \cdots \alpha_{d} \beta_{d}$ of $T_{1}$ is uniform. This is a contradiction. Hence $\Gamma$ does not contain a cycle.

Since $\Gamma$ does not contain a cycle, the graph $\Gamma$ is a forest with no-isolated vertices. This verifies at once our next claim

Claim 4. There are at least two leaves in $\Gamma$.

Set $\mathcal{T}=\left\{T_{1}, \ldots, T_{k}\right\}$,

$$
\mathcal{S}_{1}=\bigcup_{i=1}^{r} \operatorname{Supp} X_{i} \quad \text { and } \quad \mathcal{S}_{2}=\bigcup_{i=1}^{s} \operatorname{Supp} Y_{i} .
$$

For $i=1$, 2, let $a_{i}=\left|\mathcal{T} \backslash \mathcal{S}_{i}\right|$. Suppose without loss of generality that $X_{1}$ has valency one in $\Gamma$. Since $X_{1}$ is a non-trivial strip, $\left|\operatorname{Supp} X_{1}\right| \geqslant 2$, and hence there must be some $T_{i} \in \operatorname{Supp} X_{1}$ that is not covered by a strip in $Y$. Assume without loss of generality that $i=1$. This implies that $T_{1} \in \mathcal{T} \backslash \mathcal{S}_{2}$, and so $a_{2}=\left|\mathcal{T} \backslash \mathcal{S}_{2}\right| \geqslant 1$.

Claim 5. Some $Y_{i}$ has valency one, and hence $a_{1} \geqslant 1$ as well as $a_{2} \geqslant 1$.

Proof of Claim 5. Suppose to the contrary that every $Y_{i}$ has valency at least two. Then a second strip of $X, X_{2}$ say, also has valency 1 , so some $T_{i} \in \operatorname{Supp} X_{2}$ with $i \geqslant 2$ also lies in $\mathcal{T} \backslash \mathcal{S}_{2}$. Let $Y_{s+1}$ be a full strip such that $\operatorname{Supp} Y_{s+1}=\mathcal{T} \backslash \mathcal{S}_{2}$. Since $\left|\mathcal{T} \backslash \mathcal{S}_{2}\right| \geqslant 2$, the strip $Y_{s+1}$ is non-trivial. Set $\bar{Y}=Y \times Y_{s+1}$. Since $T^{k}=X Y$, we have $T^{k}=X \bar{Y}$. However, the graph that corresponds to the factorisation $T^{k}=X \bar{Y}$ has no vertices of valency 1 , which contradicts Claim 4 applied to the graph corresponding to the factorisation $T^{k}=X \bar{Y}$. Thus $Y_{i}$ has valency one for some $i$, and hence $a_{1} \geqslant 1$ also.

Claim 6. $a_{1}=a_{2}=1$.

Proof of Claim 6. If $a_{1} \geqslant 2$ and $a_{2} \geqslant 2$, then let $X_{r+1}$ and $Y_{s+1}$ be full strips such that $\operatorname{Supp} X_{r+1}=\mathcal{T} \backslash \mathcal{S}_{1}$ and $\operatorname{Supp} Y_{s+1}=\mathcal{T} \backslash \mathcal{S}_{2}$. Since $a_{1}, a_{2} \geqslant 2, X_{r+1}$ and $Y_{s+1}$ are non-trivial strips. Set $\bar{X}=X \times X_{r+1}$ and $\bar{Y}=Y \times Y_{s+1}$. Since $T^{k}=X Y$, we have $T^{k}=\bar{X} \bar{Y}$. However, the graph that corresponds to the factorisation $T^{k}=\bar{X} \bar{Y}$ has no vertex of valency one, which contradicts Claim 4 applied to the graph of the factorisation $T^{k}=\bar{X} \bar{Y}$.

Thus $\min \left\{a_{1}, a_{2}\right\}=1$, and without loss of generality we may assume that $a_{1}=1$. If $a_{2} \geqslant 2$, then we may construct $Y_{s+1}$ and $\bar{Y}$ as in the proof of Claim 5. The graph that corresponds to the factorisation $T^{k}=X \bar{Y}$ has only one vertex of valency one contradicting Claim 4.

Let us now obtain a final contradiction. It follows from Claims 4 and 6 that there is exactly one strip in $X$ with valency 1 and exactly one such strip in $Y$. All other strips have valency at least 2 . A forest with precisely two leaves is a path. Hence $\Gamma$ is a path of the form

$$
X_{1}-Y_{1}-\cdots-X_{r}-Y_{r}
$$


The valencies of $X_{1}$ and of $Y_{r}$ are equal to one. Further, the valency of each of $Y_{1}, X_{2}, \ldots, X_{r}$ is equal to two. Hence $\left|\operatorname{Supp} X_{i}\right|=\left|\operatorname{Supp} Y_{i}\right|=2$ for all $i$. Let

$$
z=\left|\left\{\left(X_{i}, T_{j}\right) \mid T_{j} \in \operatorname{Supp} X_{i}\right\}\right|
$$

Since $\left|\operatorname{Supp} X_{i}\right|=2$ for all $i$, we have $z=2 r$. On the other hand, by Claim 6 , there exists a unique $j_{0}$ such that $T_{j_{0}} \notin \bigcup_{i} \operatorname{Supp} X_{i}$ and if $j \neq j_{0}$ then there is a unique $X_{i}$ such that $T_{j} \in \operatorname{Supp} X_{i}$. Thus $2 r=z=k-1$ and so $k=2 r+1$. By possibly reordering the $T_{i}$, we may assume that there exist, for $i=1, \ldots, r$, isomorphisms $\alpha_{i}: T_{2 i-1} \rightarrow T_{2 i}$ and $\beta_{i}: T_{2 i} \rightarrow T_{2 i+1}$ such that

$$
\begin{aligned}
& X=\left\{\left(t_{1}, t_{1} \alpha_{1}, t_{2}, t_{2} \alpha_{2}, \ldots, t_{r}, t_{r} \alpha_{r}, 1\right) \mid t_{i} \in T_{2 i-1}\right\} \text { and } \\
& Y=\left\{\left(1, s_{1}, s_{1} \beta_{1}, s_{2}, s_{2} \beta_{2}, \ldots, s_{r}, s_{r} \beta_{r}\right) \mid s_{i} \in T_{2 i}\right\} .
\end{aligned}
$$

Suppose that $x \in T_{1}$. Then there are $t_{i} \in T_{2 i-1}$ and $s_{i} \in T_{2 i}$ such that $(x, 1, \ldots, 1)$ equals

$$
\left(t_{1}^{-1}, t_{1}^{-1} \alpha_{1}, t_{2}^{-1}, t_{2}^{-1} \alpha_{2}, \ldots, t_{r}^{-1}, t_{r}^{-1} \alpha_{r}, 1\right) \cdot\left(1, s_{1}, s_{1} \beta_{1}, s_{2}, s_{2} \beta_{2}, \ldots, s_{r}, s_{r} \beta_{r}\right) .
$$

Comparing the entries from the $k$-th to the 1 -st in order, we obtain that $s_{i}=t_{i}=1$ for all $i$, which is a contradiction if $x \neq 1$. Thus Theorem 1.2 is proved.

The following example shows, for a group $G$ that admits uniform automorphisms with some additional properties, that the direct product $G^{k}$ may admit factorisations with subgroups that involve longer strips.

Example 2.5. Consider a group $G$. We wish to factorise $G^{6}$ as $G^{6}=X Y$ where $X$ is a direct product of two strips of length three and $Y$ is a direct product of three strips of length two. Suppose that there exist uniform automorphisms $\alpha_{2}, \alpha_{3}$ of $G$ such that for all $y_{1}, y_{2} \in G$ there exists $t$ in $G$ such that both $t^{-1}\left(t \alpha_{2}\right)=y_{1}$ and $t^{-1}\left(t \alpha_{3}\right)=y_{2}$. In other words, the map $G \rightarrow G \times G$ defined by $t \mapsto\left(t^{-1}\left(t \alpha_{2}\right), t^{-1}\left(t \alpha_{3}\right)\right)$ is surjective. If $G$ is a non-trivial finite group, then $|G \times G|>|G|$, and hence such automorphisms do not exist for finite $G$.

Set

$$
\begin{aligned}
& X=\{(t, t, t, s, s, s) \mid t, s \in G\} \\
& Y=\left\{\left(t_{1}, t_{2}, t_{3}, t_{1}, t_{2} \alpha_{2}, t_{3} \alpha_{3}\right) \mid t_{i} \in G\right\} .
\end{aligned}
$$

We claim that $G^{6}=X Y$. Indeed, let $\left(x_{1}, \ldots, x_{6}\right) \in G^{6}$. Choose $t$ in $G$ such that $t\left(t^{-1} \alpha_{2}\right)=x_{1} x_{4}^{-1} x_{5}\left(x_{2}^{-1} \alpha_{2}\right)$ and $t\left(t^{-1} \alpha_{3}\right)=x_{1} x_{4}^{-1} x_{6}\left(x_{3}^{-1} \alpha_{3}\right)$. Let $t_{1} \in G$ such that $t t_{1}=x_{1}$. Then it follows by the assumptions above that

$$
\left(t, t, t, x_{4} t_{1}^{-1}, x_{4} t_{1}^{-1}, x_{4} t_{1}^{-1}\right) \cdot\left(t_{1}, t^{-1} x_{2}, t^{-1} x_{3}, t_{1},\left(t^{-1} x_{2}\right) \alpha_{2},\left(t^{-1} x_{3}\right) \alpha_{3}\right)=\left(x_{1}, \ldots, x_{6}\right) .
$$

Therefore $G^{6}=X Y$.

At the time of writing, we do not know if there exists an infinite simple group admitting a pair of automorphisms as in Example 2.5, and hence we state the following problem. 
Problem 2.6. Exhibit a group $G$ that admits a pair $(\alpha, \beta)$ of automorphisms such that the map $G \rightarrow G \times G$ defined by $g \mapsto\left(g^{-1}(g \alpha), g^{-1}(g \beta)\right)$ is surjective; or prove that no such group exists.

\section{ABstract CARTESIAN FACTORISATIONS INVOLVING STRIPS}

A characteristically simple group $M$ is said to be finitely completely reducible (FCR) if it is isomorphic to a the direct product $T^{k}$ for a simple group $T$ and for an integer $k \geqslant 1$. A finite characteristically simple group is FCR. Suppose that $M$ is a group and $\mathcal{K}=\left\{K_{1}, \ldots, K_{\ell}\right\}$ is a family of proper subgroups of $M$. Then $\mathcal{K}$ is said to be an abstract cartesian factorisation of $M$ if

$$
M=K_{i}\left(\bigcap_{j \neq i} K_{j}\right) \quad \text { for all } \quad i \in\{1, \ldots, \ell\}
$$

the subgroups $K_{i}$ of an abstract cartesian factorisation are called cartesian factors of $M$.

Cartesian factorisations were introduced in [BPS04], where they were called 'cartesian systems of subgroups', to characterise cartesian decompositions preserved by innately transitive groups. In this section we investigate cartesian factorisations in characteristically simple FCR-groups in which the cartesian factors involve strips. Let us introduce notation for this section. Let $M=T_{1} \times \cdots \times T_{k}$ be a characteristically simple FCRgroup with simple normal subgroups $T_{1}, \ldots, T_{k}$, and let $G_{0}$ be a subgroup of Aut $M$ such that the natural action of $G_{0}$ on $T_{1}, \ldots, T_{k}$ is transitive. Suppose, in addition, that $\mathcal{K}=\left\{K_{1}, \ldots, K_{\ell}\right\}$ is an abstract $G_{0}$-invariant cartesian factorisation of $M$. Set $M_{0}=\bigcap_{i} K_{i}$. If $T$ is abelian, then $T$ is cyclic of prime order $p$, and $M$ can be considered as a finite-dimensional vector space over $\mathbb{F}_{p}$. In this case a cartesian factorisation of $M$ is essentially a direct sum decomposition. Therefore we assume that $M$ is non-abelian.

Recall from Section 2 that if $I$ is a subset of $\mathcal{T}=\left\{T_{1}, \ldots, T_{k}\right\}$ or a subset of $\{1, \ldots, k\}$, then $\sigma_{I}$ denotes the corresponding coordinate projection from $M$ onto either $\prod_{T_{i} \in I} T_{i}$ or onto $\prod_{i \in I} T_{i}$, respectively.

A strip $X$ is involved in a subgroup $K$ of $M$ if $K=X \times K \sigma_{\mathcal{T} \backslash \operatorname{Supp} X}$ where $\mathcal{T}=\left\{T_{1}, \ldots, T_{k}\right\}$. We say that a strip $X$ is involved in a cartesian factorisation $\mathcal{K}$ if $X$ is involved in a member $K \in \mathcal{K}$. In this case, (3) implies that $X$ is involved in a unique member of $\mathcal{K}$. Uniform automorphisms were introduced in Section 2. In this section we prove the following theorem.

Proposition 3.1. Suppose that $\mathcal{K}=\left\{K_{1}, \ldots, K_{\ell}\right\}, M=T_{1} \times \cdots \times T_{k}, M_{0}$, and $G_{0}$ are as above and let $X_{1}$ and $X_{2}$ be two non-trivial full strips involved in $\mathcal{K}$ such that Supp $X_{1} \cap \operatorname{Supp} X_{2} \neq \emptyset$. Then the following both hold:

(1) $T_{1}$ admits a uniform automorphism;

(2) $M_{0}$ is not a subdirect subgroup of $M$. 
Proof. The proof of this theorem is quite involved, and so we split it into a series of claims. Assume that the hypotheses of Proposition 3.1 hold. Assume, moreover, that either $T_{1}$ does not admit a uniform automorphism, or that $M_{0}$ is a subdirect subgroup of $M$.

Claim 1. $\left|\operatorname{Supp} X_{1} \cap \operatorname{Supp} X_{2}\right|=1$.

Proof of Claim 1. By the definition of 'being involved' for strips, if $X_{1}, X_{2}$ are involved in the same $K_{i}$ then they are disjoint as strips. Thus we may assume without loss of generality that $X_{1}$ is involved in $K_{1}$ and $X_{2}$ is involved in $K_{2}$. Suppose to the contrary that $T_{1}, T_{2} \in \operatorname{Supp} X_{1} \cap \operatorname{Supp} X_{2}$ and set $\sigma=\sigma_{\{1,2\}}$. Then $Y_{1}=K_{1} \sigma$ and $Y_{2}=K_{2} \sigma$ are non-trivial full strips in $T_{1} \times T_{2}$ such that $Y_{1} Y_{2}=M \sigma=T_{1} \times T_{2}$. By Lemma 2.3, $T_{1}$ admits a uniform automorphism. Thus, by our own assumption, $M_{0}$ is a subdirect subgroup of $M$. Then $K_{1} \cap K_{2}$ is also a subdirect subgroup of $M$, and so $\left(K_{1} \cap K_{2}\right) \sigma$ is a subdirect subgroup in $T_{1} \times T_{2}$. Now $Y_{1}=\left\{(t, t \alpha) \mid t \in T_{1}\right\}$ and $Y_{2}=\left\{(t, t \beta) \mid t \in T_{1}\right\}$ where $\alpha, \beta: T_{1} \rightarrow T_{2}$ are isomorphisms. Then

$$
\left(K_{1} \cap K_{2}\right) \sigma \leqslant Y_{1} \cap Y_{2}=\left\{(t, t \alpha) \mid t \in T_{1} \text { such that } t \alpha=t \beta\right\} .
$$

Now the fact that $\left(K_{1} \cap K_{2}\right) \sigma$ is subdirect in $T_{1} \times T_{2}$ implies that $t \alpha=t \beta$ for all $t \in T_{1}$, and hence $\alpha=\beta$. However, this implies that $Y_{1}=Y_{2}$, yielding $Y_{1} Y_{2} \neq T_{1} \times T_{2}$. This contradiction proves the claim.

Claim 2. There exists a sequence of strips $X_{1}, \ldots, X_{a}$, with $a \geqslant 3$, involved in $\mathcal{K}$ such that

(1) $X_{1}$ and $X_{j}$ are disjoint for $j \notin\{a, 2\}$;

(2) for $i=2, \ldots, a-1$ and $j \notin\{i-1, i+1\}$, the strips $X_{i}$ and $X_{j}$ are disjoint;

(3) $X_{a}$ and $X_{j}$ are disjoint for $j \notin\{a-1,1\}$;

(4) and finally,

$$
\begin{aligned}
\left|\operatorname{Supp} X_{1} \cap \operatorname{Supp} X_{2}\right|=\mid \operatorname{Supp} X_{2} & \cap \operatorname{Supp} X_{3} \mid=\cdots \\
= & \left|\operatorname{Supp} X_{a-1} \cap \operatorname{Supp} X_{a}\right|=\left|\operatorname{Supp} X_{a} \cap \operatorname{Supp} X_{1}\right|=1 .
\end{aligned}
$$

Proof of Claim 2. By Claim 1, Supp $X_{1} \cap \operatorname{Supp} X_{2}=\left\{T_{t}\right\}$ for some $t \leqslant k$. Choose $g \in G_{0}$ such that $T_{t}^{g} \in \operatorname{Supp} X_{2} \backslash \operatorname{Supp} X_{1}$; such an element $g$ exists since $G_{0}$ is transitive on $\mathcal{T}=\left\{T_{1}, \ldots, T_{k}\right\}$ and Supp $X_{2} \backslash \operatorname{Supp} X_{1}$ is non-empty. Now $G_{0}$ acts by conjugation on the set of full strips involved in $\mathcal{K}$, and so both $X_{1}^{g}$ and $X_{2}^{g}$ are full strips involved in $\mathcal{K}$. As $T_{t}^{g}$ is in both Supp $X_{1}^{g}$ and $\operatorname{Supp} X_{2}^{g}$, but is not in $\operatorname{Supp} X_{1}$ we deduce that there exists a nontrivial strip $X_{3}$ in $\mathcal{K}$ distinct from $X_{1}, X_{2}$ such that $\operatorname{Supp} X_{3} \cap\left(\operatorname{Supp} X_{2} \backslash \operatorname{Supp} X_{1}\right) \neq \emptyset$ (namely, we can take $X_{3}$ to be one of $X_{1}^{g}$ or $X_{2}^{g}$ as at least one of these is distinct from $X_{1}$ and $X_{2}$ ). Proceeding in this way we construct a sequence $X_{1}, X_{2}, \ldots$ of distinct, nontrivial strips in $\mathcal{K}$ such that $\operatorname{Supp} X_{d+1} \cap\left(\operatorname{Supp} X_{d} \backslash \operatorname{Supp} X_{d-1}\right) \neq \emptyset$ for each $d \geqslant 2$. Since $k$ is finite, there exists $a$ such that

$$
\operatorname{Supp} X_{a} \cap\left(\operatorname{Supp} X_{1} \cup \cdots \cup \operatorname{Supp} X_{a-2}\right) \neq \emptyset \text {. }
$$


Let $a$ be the least integer such that this property holds. The conditions imposed on $X_{1}$ and $X_{2}$ imply that $a \geqslant 3$. By removing some initial segment of the sequence and relabelling the $X_{i}$ if necessary, we may assume that the intersection Supp $X_{a} \cap \operatorname{Supp} X_{1}$ is non-empty, while Supp $X_{a} \cap \operatorname{Supp} X_{d}=\emptyset$ if $2 \leqslant d \leqslant a-2$ for some $a \geqslant 3$. Now, applying Claim 1 a number of times, the sequence $X_{1}, \ldots, X_{a}$ is as required.

Now assume that the conditions of Claim 2 are valid, $X_{1}$ and $X_{2}$ are non-disjoint strips involved in $\mathcal{K}$ and select $X_{1}, \ldots, X_{a}$ as in the proof of Claim 2. By relabelling the $K_{i}$ we may assume that $X_{1}$ is involved in $K_{1}$. Let $1=i_{1}<i_{2}<\cdots<i_{d}<a$ be such that among the $X_{i}$ the strips $X_{i_{1}}, \ldots, X_{i_{d}}$ are precisely the ones that are involved in $K_{1}$. Note that $X_{a}$ is not involved in $K_{1}$ since $\operatorname{Supp} X_{a}$ and $\operatorname{Supp} X_{1}$ are not disjoint. Also, $i_{j+1} \geqslant i_{j}+2$ for all $j=1, \ldots, d-1$ since $\operatorname{Supp} X_{i_{j}}$ and $\operatorname{Supp} X_{i_{j}+1}$ are not disjoint. We may also relabel the $T_{i}$ so that

$$
\left\{T_{1}\right\}=\operatorname{Supp} X_{a} \cap \operatorname{Supp} X_{1} \quad \text { and } \quad\left\{T_{2}\right\}=\operatorname{Supp} X_{1} \cap \operatorname{Supp} X_{2},
$$

and so that for $j=2, \ldots, d$,

$$
\begin{aligned}
\left\{T_{2 j-1}\right\} & =\operatorname{Supp} X_{i_{j}-1} \cap \operatorname{Supp} X_{i_{j}} \\
\left\{T_{2 j}\right\} & =\operatorname{Supp} X_{i_{j}} \cap \operatorname{Supp} X_{i_{j}+1}
\end{aligned}
$$

It follows from Claim 2, that $T_{1}, \ldots, T_{2 d}$ are pairwise distinct. Define the projection map

$$
\sigma: M \rightarrow T_{1} \times \cdots \times T_{2 d} \text { and } \widehat{K_{1}}=\bigcap_{i \neq 1} K_{i}
$$

Claim 3. Using the notation introduced above, the following hold.

(1) $K_{1} \sigma$ is a direct product $Y_{1} \times \cdots \times Y_{d}$ such that each $Y_{i}$ is a non-trivial full strip in $T_{2 i-1} \times T_{2 i}$ and $Y_{i}=\left\{\left(t, t \alpha_{i}\right) \mid t \in T_{2 i-1}\right\}$ for some isomorphism $\alpha_{i}: T_{2 i-1} \rightarrow T_{2 i}$.

(2) $\widehat{K}_{1} \leqslant Z_{1} \times \cdots \times Z_{d}$, such that, for $i=1, \ldots, d-1, Z_{i}=\left\{\left(t, t \beta_{i}\right) \mid t \in T_{2 i}\right\}$ is a nontrivial full strip in $T_{2 i} \times T_{2 i+1}$ where $\beta_{i}: T_{2 i} \rightarrow T_{2 i+1}$ is an isomorphism. Further $Z_{d}=\left\{\left(t \beta_{d}, t\right) \mid t \in T_{2 d}\right\}$ is a non-trivial full strip in $T_{2 d} \times T_{1}$ where $\beta_{d}: T_{2 d} \rightarrow T_{1}$ is an isomorphism.

Proof of Claim 3. Assertion (1) follows from the observation that

$$
K_{1} \sigma=\left(X_{i_{1}} \times \cdots \times X_{i_{d}}\right) \sigma=Y_{1} \times \cdots \times Y_{d} \quad \text { with } \quad Y_{j}=X_{i_{j}} \sigma \text { for each } j .
$$

Let us prove assertion (2). It suffices to show that $\widehat{K_{1}} \sigma_{\{2 i, 2 i+1\}} \leqslant Z_{i}$ for $i=1, \ldots, d-1$ and $\widehat{K_{1}} \sigma_{\{1,2 d\}} \leqslant Z_{d}$. We prove the claim for $i=1$, that is, for $\widehat{K_{1}} \sigma_{\{2,3\}}$, noting that the proof for the other projections is identical. Set $r=i_{2}-1$. Then the strips $X_{2}, \ldots, X_{r}$ are 'between' $X_{1}$ and $X_{i_{2}}$ in the sequence of the $X_{i}$ and they are not involved in $K_{1}$. Choose $T_{m_{1}}, \ldots, T_{m_{r}}$ such that $\left\{T_{m_{i}}\right\}=\operatorname{Supp} X_{i} \cap \operatorname{Supp} X_{i+1}$. By the choice made earlier, we have $T_{m_{1}}=T_{2}$ and $T_{m_{r}}=T_{3}$. Let $\sigma^{\prime}$ denote the projection onto $T_{m_{1}} \times \cdots \times T_{m_{r}}$. By Claim 2, the indices $m_{1}, \ldots, m_{r}$ are pairwise distinct. Suppose that $x=\left(t_{m_{1}}, \ldots, t_{m_{r}}\right)$ is an element of $\widehat{K_{1}} \sigma^{\prime}$ with $t_{i} \in T_{m_{i}}$. The strip $X_{2}$ is 
involved in $K_{m}$ for some $m \neq 1$. Recall that $X_{2}$ covers $T_{m_{1}}=T_{2}$ and $T_{m_{2}}$. Then $x \in K_{m} \sigma^{\prime}$ and hence $\left(t_{m_{1}}, t_{m_{2}}\right) \in X_{2} \sigma_{\left\{m_{1}, m_{2}\right\}}$. Thus there exists an isomorphism $\gamma_{m_{1}}: T_{m_{1}} \rightarrow T_{m_{2}}$ such that $X_{2} \sigma_{\left\{m_{1}, m_{2}\right\}}=\left\{\left(t, t \gamma_{m_{1}}\right) \mid t \in T_{m_{1}}\right\}$. Using the same argument, we find that there exist isomorphisms $\gamma_{m_{i}}: T_{m_{i}} \rightarrow T_{m_{i+1}}$, for $i=1, \ldots, r-1$, such that $X_{i+1} \sigma_{\left\{m_{i}, m_{i+1}\right\}}=\left\{\left(t, t \gamma_{m_{2}}\right) \mid t \in T_{m_{i}}\right\}$. Thus $t_{m_{r}}=t_{m_{1}} \gamma_{m_{1}} \cdots \gamma_{m_{r-1}}$. Set $\beta_{1}=\gamma_{m_{1}} \cdots \gamma_{m_{r-1}}$. This argument shows that

$$
\widehat{K_{1}} \sigma_{\{2,3\}} \leqslant\left\{\left(t, t \beta_{1}\right) \mid t \in T_{2}\right\}:=Z_{1} .
$$

Therefore $\widehat{K_{1}} \sigma_{\{2,3\}} \leqslant Z_{1}$. The proof for the other projections is identical. This shows that assertion (2) holds.

Claim 4. Use the notation of Claim 3, and set $\alpha=\alpha_{1} \beta_{1} \alpha_{2} \cdots \alpha_{d} \beta_{d}$. Then the following hold.

(1) $\alpha \in$ Aut $T_{1}$ and $\alpha$ is uniform.

(2) $M_{0}$ is not a subdirect subgroup of $M$ where $M_{0}=\bigcap_{i} K_{i}$.

Proof of Claim 4. (1) It follows from Claim 3 that $\alpha \in$ Aut $T_{1}$. Since $K_{1} \widehat{K_{1}}=M$, we have $\left(K_{1} \sigma\right)\left(\widehat{K_{1}} \sigma\right)=T_{1} \times \cdots \times T_{2 d}$ with $\sigma$ and $\widehat{K}_{1}$ as in (4). Therefore

$$
\left(Y_{1} \times \cdots \times Y_{d}\right)\left(Z_{1} \times \cdots \times Z_{d}\right)=T_{1} \times \cdots \times T_{2 d} .
$$

Since the factorisation in (5) is as in Lemma 2.4, it follows from Lemma 2.4 that $\alpha$ is uniform.

(2) By definition, $K_{1} \cap \widehat{K_{1}}=M_{0}$. Suppose that $M_{0}$ is subdirect in $M$. Then $M_{0} \sigma$ is also a subdirect subgroup of $T_{1} \times \cdots \times T_{2 d}$. Suppose that $x=\left(t_{1}, t_{2}, \ldots, t_{2 d}\right) \in M_{0} \sigma$. Then $x \in K_{1} \sigma$ and $x \in \widehat{K_{1}} \sigma$, and so $t_{2 i}=t_{2 i-1} \alpha_{i}$, for $i=1, \ldots, d$, and also $t_{2 i+1}=t_{2 i} \beta_{i}$ for $i=1, \ldots, d-1$, and $t_{1}=t_{d} \beta_{d}$. Thus $t_{1}=t_{1} \alpha_{1} \beta_{1} \cdots \alpha_{d} \beta_{d}=t_{1} \alpha$. Since $M_{0} \sigma$ is subdirect, this has to hold for all $t_{1} \in T_{1}$, and hence $\alpha=1$. However, by part (1), $\alpha$ is uniform, which is a contradiction, as the identity automorphism is not uniform.

Now Proposition 3.1 follows at once from Claim 4.

\section{QuAsiprimitive GROUPS OF DIAGONAL TYPE}

Recall that a permutation group acting on $\Omega$ is quasiprimitive if all the non-trivial normal subgroups of $G$ are transitive. A quasiprimitive group $G$ is said to be of diagonal type if it has a unique minimal normal FCR subgroup $M$ such that $M=T_{1} \times \cdots \times T_{k}$ where the $T_{i}$ are non-abelian simple groups and a point stabiliser $M_{\alpha}$ is a subdirect subgroup of $M$; that is, denoting the coordinate projections by $\sigma_{i}, M_{\alpha} \sigma_{i}=T_{i}$ for all $i$. By Scott's Lemma [Sco80, Lemma, page 328], we have, in such a quasiprimitive group of diagonal type, that $M_{\alpha}$ is a direct product of pairwise disjoint full strips ${ }^{1}$. The group $G$ is said to

\footnotetext{
${ }^{1}$ Scott's Lemma is most often applied to finite simple groups, but it holds for infinite simple groups and the proof presented in [Sco80] does not assume finiteness.
} 
have simple diagonal type if $M_{\alpha}$ is simple (that is, $M_{\alpha}$ is a full strip), and otherwise $G$ is said to have compound diagonal type.

If $\Gamma$ is a set and $\ell \geqslant 2$, then the wreath product Sym $\Gamma \ell S_{\ell}$ can be considered as a permutation group on $\Gamma^{\ell}$ in its product action, which is defined as

$$
\left(\gamma_{1}, \ldots, \gamma_{\ell}\right)\left(g_{1}, \ldots, g_{\ell}\right) \sigma=\left(\gamma_{1 \sigma^{-1}} g_{1 \sigma^{-1}}, \ldots, \gamma_{\ell \sigma^{-1}} g_{\ell \sigma^{-1}}\right)
$$

for all $\gamma_{1}, \ldots, \gamma_{\ell} \in \Gamma, g_{1}, \ldots, g_{\ell} \in \operatorname{Sym} \Gamma$, and $\sigma \in \mathbf{S}_{\ell}$.

Theorem 4.1. Let $G$ be a quasiprimitive group of diagonal type acting on $\Omega$ with minimal normal subgroup $M=T_{1} \times \cdots \times T_{k}$ where the $T_{i}$ are non-abelian simple groups. Then $G$ can be embedded into a subgroup $W$ of $\Omega$ that is permutationally isomorphic to $\mathrm{Sym} \boldsymbol{\imath} \mathrm{S}_{\ell}$ in product action with $|\Gamma| \geqslant 2$ and $\ell \geqslant 2$ if and only if $G$ is of compound diagonal type.

Proof. We suppose that $G$ is quasiprimitive of diagonal type with a minimal normal subgroup $M$ as in the statement. Suppose that there exists a subgroup $W$ of Sym $\Omega$ that is permutationally isomorphic to $\operatorname{Sym} \Gamma \ell \mathrm{S}_{\ell}$ with $|\Gamma| \geqslant 2$ and $\ell \geqslant 2$ such that $G \leqslant W$. Then $\Omega$ can be identified with $\Gamma^{\ell}$, and so from now on we assume that $\Omega=\Gamma^{\ell}$ and that $W=\operatorname{Sym} \Gamma \imath \mathrm{S}_{\ell}$. Note that $M$ is transitive on $\Omega$, since $G$ is quasiprimitive.

Let $\pi: W \rightarrow \mathrm{S}_{\ell}$ denote the natural projection. Since $M$ is a minimal normal subgroup of $G$, either $M \leqslant \operatorname{ker} \pi$ or $M \cap \operatorname{ker} \pi=1$. In the latter case, the restriction of $\pi$ to $M$ is a faithful permutation representation of $M$ and hence $M$ is isomorphic to a subgroup of $\mathrm{S}_{\ell}$. This implies that $M$ is finite and, since $M$ is transitive on $\Omega$, the sets $\Omega$ and $\Gamma$ are finite. Now if $p$ is a prime dividing $|\Gamma|$, then $p^{\ell}|| \Omega \mid$, and hence, as $M$ is transitive on $\Omega$, $p^{\ell}|| M \mid$, which gives $p^{\ell} \mid \ell$ !. Since this is impossible (see [JJ98, Exercise 2.20]), we must have $M \leqslant \operatorname{ker} \pi$. Therefore $M$ is contained in the base group $B=(\operatorname{Sym} \Gamma)^{\ell}$ of $W$.

The action of the base group can be viewed via $\ell$ projection maps $\pi_{1}, \ldots, \pi_{\ell}: B \rightarrow \operatorname{Sym} \Gamma$ given by $\left(g_{1}, \ldots, g_{\ell}\right) \pi_{i}=g_{i}$ for $\left(g_{1}, \ldots, g_{\ell}\right) \in(\operatorname{Sym} \Gamma)^{\ell}$. In this way we may write, for $g \in B$ and $\left(\gamma_{1}, \ldots, \gamma_{\ell}\right) \in \Omega$, that

$$
\left(\gamma_{1}, \ldots, \gamma_{\ell}\right) g=\left(\gamma_{1}\left(g \pi_{1}\right), \ldots, \gamma_{\ell}\left(g \pi_{\ell}\right)\right)
$$

In particular, the last equation is valid if $g \in M$. We consider the homomorphisms $\pi_{1}, \ldots, \pi_{\ell}$ as permutation representations of $B$. Choose $\gamma \in \Gamma$, set $\omega=(\gamma, \ldots, \gamma) \in \Omega$, and, for $i=1, \ldots, \ell$, let $K_{i}$ denote the stabiliser in $M$ of $\gamma$ under $\pi_{i}$. Since $M$ is transitive on $\Omega$, each $M \pi_{i}$ is transitive on $\Gamma$, and, since $|\Gamma| \geqslant 2$, each $K_{i}$ is a proper subgroup of $M$.

We claim that the set $\mathcal{K}=\left\{K_{1}, \ldots, K_{\ell}\right\}$ of proper subgroups of $M$ is an abstract cartesian factorisation of $M$. Let us prove that $M=K_{1}\left(\bigcap_{i \neq 1} K_{i}\right)$; the other factorisations in equation (3) can be shown similarly. Setting $\bar{K}_{1}=\bigcap_{i \neq 1} K_{i}$ and noting that $K_{1}$ is the point stabiliser in $M$ under its transitive action on $\Gamma$ by $\pi_{1}$, the factorisation $M=K_{1} \bar{K}_{1}$ is equivalent to the assertion that $\bar{K}_{1}$ is transitive on $\Gamma$ under the representation $\pi_{1}$. Suppose that $\gamma^{\prime} \in \Gamma$ and consider the point $\omega^{\prime}=\left(\gamma^{\prime}, \gamma, \ldots, \gamma\right)$. Since $M$ is transitive on $\Omega$, there exists some $m \in M$ such that $\omega m=\omega^{\prime}$. Considering the definitions of $\omega$ and $\omega^{\prime}$, this implies that $\gamma\left(m \pi_{1}\right)=\gamma^{\prime}$ and $\gamma\left(m \pi_{i}\right)=\gamma$ for all $i=2, \ldots, \ell$; that is, $m \in \bar{K}_{1}$. Since $\gamma^{\prime}$ is chosen arbitrarily, $\bar{K}_{1}$ is transitive on $\Gamma$ under $\pi_{1}$, and so $K_{1} \bar{K}_{1}=M$, as claimed. As 
noted above, the other factorisations of (3) follow similarly, and $\mathcal{K}$ is an abstract cartesian factorisation for $M$, as claimed.

Since $\bigcap_{i \geqslant 1} K_{i}$ is the intersections of the stabilisers of $\gamma$ under the representations $\pi_{1}, \ldots, \pi_{\ell}$ and $\omega=(\gamma, \ldots, \gamma)$, it follows that

$$
\bigcap_{i=1}^{\ell} K_{i}=M_{\omega} .
$$

Further, $G_{\omega}$ permutes by conjugation the $\ell$ direct factors of the base group $B$, and so the set $\mathcal{K}$ is invariant under conjugation by $G_{\omega}$.

Since $M$ is a minimal normal subgroup of $G, G$ is transitive by conjugation on the $T_{i}$, and so the $T_{i}$ are pairwise isomorphic. Let $T$ denote the common isomorphism type of the $T_{i}$. Since $G$ has diagonal type, $M_{\omega}$ is a subdirect subgroup of $M$. For each $K \in \mathcal{K}$ we have $M_{\omega} \leqslant K$, and so all elements of $\mathcal{K}$ are subdirect subgroups of $M$. Let $K_{1}, K_{2} \in \mathcal{K}$ be distinct subgroups. Then $K_{1}, K_{2} \neq M$, and so, $K_{1}, K_{2}$ involve non-trivial full strips $X_{1}$ and $X_{2}$, say, and, by the factorisation in equation (3), $M=K_{1} K_{2}$, which implies $X_{1} \neq X_{2}$. By Proposition 3.1, $X_{1}$ and $X_{2}$ are disjoint strips. This means, in particular, that if $X$ is a non-trivial full strip involved in $K_{j}$ covering $T_{i}$, then $T_{i} \leqslant K_{m}$ for all $m \neq j$. Therefore if $X_{1}, \ldots, X_{s}$ are the non-trivial full strips involved in $\mathcal{K}$, then $M_{\omega}=X_{1} \times \cdots \times X_{s}$. Further, since each $K \in \mathcal{K}$ involve at least one non-trivial full strip and $|\mathcal{K}| \geqslant 2$, the argument above shows that $s \geqslant 2$. Hence $M_{\omega}$ is a direct product of at least two non-trivial full strips, and so $G$ has compound diagonal type.

Conversely, suppose that $G$ has compound diagonal type, and so $M_{\omega}$ is a direct product $M_{\omega}=X_{1} \times \cdots \times X_{r}$ of non-trivial full strips $X_{i}$. Then $M$ admits a non-trivial direct product decomposition $M=M_{1} \times \cdots \times M_{r}$ such that

$$
M_{\omega}=\left(M_{1} \cap M_{\omega}\right) \times \cdots \times\left(M_{r} \cap M_{\omega}\right) .
$$

Setting $\Delta$ to be the right coset space $\left[M_{1}: M_{1} \cap M_{\omega}\right]$, the transitivity of $M$ on $\Omega$ allows us to identify $\Omega$ with the direct power $\Delta^{r}$ and, under this identification, $G$ becomes a subgroup of Sym $\Delta \imath \mathrm{S}_{r}$ in product action.

Theorem 1.1 is an easy consequence of Theorem 4.1 .

\section{REFERENCES}

[BP03] Robert W. Baddeley and Cheryl E. Praeger. On primitive overgroups of quasiprimitive permutation groups. J. Algebra, 263(2):294-344, 2003.

[BPS04] Robert W. Baddeley, Cheryl E. Praeger, and Csaba Schneider. Transitive simple subgroups of wreath products in product action. J. Aust. Math. Soc., 77(1):55-72, 2004.

[BPS06] Robert W. Baddeley, Cheryl E. Praeger, and Csaba Schneider. Innately transitive subgroups of wreath products in product action. Trans. Amer. Math. Soc., 358(4):1619-1641 (electronic), 2006.

[B+94] Marcus Brazil, Jacinta Covington, Tim Penttila, Cheryl E. Praeger, and Alan R. Woods. Maximal subgroups of infinite symmetric groups. Proc. London Math. Soc. 68(3):77-111, 1994.

[CMM96] Jacinta Covington, Dugald Macpherson, and Alan Mekler. Some maximal subgroups of infinite symmetric groups. Quart. J. Math. Oxford Ser. (2) 47(187):297-311, 1996. 
[Gor13] D. Gorenstein. Finite Simple Groups: An Introduction to Their Classification. University Series in Mathematics. Springer US, 2013.

[JJ98] G. A. Jones and J. M. Jones. Elementary Number Theory. Springer Undergraduate Mathematics Series. Springer London, 1998.

[Kov88a] L. G. Kovács. Groups with uniform automorphisms. Rend. Circ. Mat. Palermo (2) Suppl., (19):125-133, 1988.

[Kov88b] L. G. Kovács. Primitive permutation groups of simple diagonal type. Israel J. Math., 63(1):119$127,1988$.

[KS06] Hans Kurzweil and Bernd Stellmacher. The Theory of Finite Groups: An Introduction. Universitext. Springer New York, 2006.

[LPS87] Martin W. Liebeck, Cheryl E. Praeger, and Jan Saxl. A classification of the maximal subgroups of the finite alternating and symmetric groups. J. Algebra, 111(2):365-383, 1987.

[MP90] H. D. Macpherson and Cheryl E. Praeger. Maximal subgroups of infinite symmetric groups. $J$. London Math. Soc. (2) 42(1):85-92, 1990.

[Pra90] Cheryl E. Praeger. The inclusion problem for finite primitive permutation groups. Proc. Lond. Math. Soc., III. Ser., 60(1):68-88, 1990.

[Sco80] Leonard L. Scott. Representations in characteristic $p$. In Bruce Cooperstein and Geoffrey Mason, editors, The Santa Cruz Conference on Finite Groups, volume 37 of Proceedings of Symposia in Pure Mathematics, pages 319-331, Providence, R.I., 1980. American Mathematical Society.

[Spr98] T. A. Springer. Linear Algebraic Groups. Progress in mathematics. Birkhäuser, 1998.

(C. E. Praeger) School of Mathematics and Statistics, The University of Western Australia, 35 Stirling Highway 6009 Crawley, Western Australia

CHERYL.PRAEGER@UWA.EDU.AU, WWW.MATHS.UWA.EDU.AU/ PRAEGER

(C. Schneider) Departamento de Matemática, Instituto de Ciências Exatas, Universidade Federal de Minas Gerais, Av. Antônio Carlos 6627, Belo Horizonte, MG, Brazil, CSABA@MAT.UFMG.BR, WWW.MAT.UFMG.BR/ CSABA 